\title{
Notas taxonômicas para Euphorbiaceae s.l. do Nordeste do Brasil ${ }^{1}$
}

\author{
Maria de Fátima de Araújo Lucena ${ }^{2,3}$ e Marccus Alves ${ }^{2}$
}

Recebido: 09.10.2008; aceito: 28.01.2010

\begin{abstract}
Taxonomic notes for Euphorbiaceae s.l. from Northeastern Brazil). Recent taxonomic studies carried out in Euphorbiaceae from the Caatinga, including field collections and surveys of 14 herbaria, have revealed 29 taxa to be added to the previously published checklist of the family in the Northeast. The taxa are presented with information on synonymy, type material and voucher specimens. Comments related to geographical distribution, habitats and diagnostic characters are also given. The study increases the diversity of species of this important family in the semi-arid region of Brazil and points out the importance of intensive sampling in new areas, as well as the analysis of botanical collections already available.

Key words: Biodiversity, dry forest, floristic, Rain Forest
\end{abstract}

RESUMO - (Notas taxonômicas para Euphorbiaceae s.l. do Nordeste do Brasil). Recentes estudos taxonômicos desenvolvidos na Caatinga, sobre Euphorbiaceae, incluindo coletas e levantamentos em 14 herbários revelaram o acréscimo de 29 táxons ao cheklist anteriormente conhecido para a família na região. Os táxons são apresentados acompanhados de alguns sinônimos, material tipo e espécimes de referência. São também apresentados comentários sobre distribuição geográfica, ambientes de ocorrência e caracteres diagnósticos. O estudo amplia a diversidade de Euphorbiaceae para o semiárido do Brasil e ressalta a importância de coletas intensivas em novas áreas, assim como a análise minuciosa dos acervos botânicos já disponíveis.

Palavras-chave: Biodiversidade, florística, Floresta Atlântica, semi-árido

\section{Introdução}

Euphorbiaceae s.l. merece destaque entre as Angiospermas por abrigar 6.300 espécies reunidas em 245 gêneros (Govaerts et al. 2000), distribuídas principalmente nas regiões tropical e subtropical, especialmente nos continentes americano e africano, com exceção para poucos gêneros extratropicais (Webster 1994). Compreende um dos grupos taxonômicos mais complexos e morfologicamente diversos entre as Eudicotiledôneas (Judd et al. 1999, Soltis et al. 2005, Simpson 2006).

No Brasil, estima-se a ocorrência de 1.100 espécies e 72 gêneros, habitando os mais diferentes tipos vegetacionais (Barroso et al. 1991, Souza \& Lorenzi
2006). Trabalho desenvolvido por Cordeiro \& Carneiro-Torres (2006) indica a ocorrência de 211 espécies e 45 gêneros para a região Nordeste do Brasil.

Euphorbiaceae s.l. compreende um grupo de plantas reunidas por Webster (1994) em cinco subfamílias, reunindo táxons uniovulados e biovulados: Phyllanthoideae, Oldfieldioideae, Acalyphoideae, Crotonoideae e Euphorbioideae. Chase et al. (2002), APG (2003), Wurdack et al. (2005) e Kathriarachchi et al. (2005) sugerem a segregação de alguns representantes em outras famílias: Phyllantaceae, Picrodendraceae, Peraceae e Putranjivaceae, reunindo nestas todos os táxons biovulados.

Registrada como importante grupo de plantas do semi-árido nordestino (Araújo et al. 1995, Alcofora-

1. Parte da Tese de Doutorado do primeiro autor no Programa de Pós-Graduação em Biologia Vegetal, Universidade Federal de Pernambuco

2. Universidade Federal de Pernambuco, Laboratório de Morfo-taxonomia Vegetal, Av. Prof. Moraes Rego s/n, Cidade Universitária, 50670-590 Recife, PE, Brasil

3. Autor para correspondência: mfaraujobotanica@yahoo.com.br 
do Filho et al. 2003, Andrade et al. 2004, Sátiro \& Roque 2008), Euphorbiaceae s.l. foi recentemente inventariada por Lucena (2009) em áreas selecionadas como prioritárias para conservação do Bioma Caatinga (MMA 2002). Este trabalho é parte do inventário realizado e objetiva contribuir com o conhecimento da família na região, através da atualização do número de táxons.

\section{Material e métodos}

Este estudo foi realizado principalmente com base em visitas a populações naturais e consultas aos principais herbários da região Nordeste (ASE, CEPEC, EAC, EAN, HST, HUEFS, IPA, JPB, MAC, MOSS, PEUFR, TEPB, UFRN, UFP) e do exterior (M, P, K) - siglas de acordo com Thiers (2009).

Coletas botânicas foram realizadas entre março de 2006 e maio de 2008, no Parque Nacional Serra da Capivara (PI), Parque Nacional Serra das Confusões (PI), Parque Nacional Serra de Itabaiana (SE), Cariris paraibanos (PB), e municípios de Mirandiba e Serra Talhada (PE) e Porto da Folha (SE), totalizando 22 expedições.

A identificação dos táxons foi realizada através de bibliografia especializada e análise de material-tipo. Os comentários apresentados foram elaborados com base nos dados contidos nas etiquetas das exsicatas, bibliografia e, especialmente, observações de campo. Foram relacionados, quando possível, os basiônimos e/ou os principais sinônimos. Os materiais de referência correspondem a exemplares examinados ou disponíveis em bibliografia de referência para o grupo taxonômico. Pelo intuito de atualizar o número de táxons, na região Nordeste, tendo como base a lista apresentada por Cordeiro \& Carneiro-Torres (2006), adotou-se Webster (1994) para classificação da família.

\section{Resultados e Discussão}

O estudo acrescenta à listagem apresentada por Cordeiro \& Carneiro-Torres (2006), 29 espécies e cinco gêneros à região Nordeste: Acalypha L. (1 sp.), Actinostemon Mart. ex Klotzsch (2 spp.), Bernardia Houst. ex Mill. (5 spp.), Cnidoscolus Pohl (4 spp.), Croton L. (6 spp.), Dalechampia Plum. ex L. (1 sp.), Discocarpus Klotzsch (1 sp.), Euphorbia L. (1 sp.), Flueggea Willd. (1 sp.), Jatropha L. (1 sp.), Meineckia Baill. (1 sp.), Philyra Klotzsch (1 sp.), Podocalyx Klotzsch (1 sp.), Tragia Plum. ex L. (2 spp.) e Savia Willd. (1 sp.). Assim, uma importante contribuição é dada à flora local acompanhada da atualização das identificações dos acervos regionais.

Entre as 29 espécies aqui apresentadas, seis são restritas à vegetação de caatinga (Cnidoscolus byssinus Fdez. Casas, C. hamosus Pohl, C. infestus Pax \& K. Hoffm., Croton acradenius Pax \& K. Hoffm., C. laceratoglandulosus Caruzo \& Cordeiro, C. lachnocladus Mart. ex Müll. Arg., Dalechampia fernandesii G.L. Webster e Jatropha hastifolia Fdez. Casas), duas ocorrem em áreas de caatinga e cerrado (Croton tenuifolius Pax \& K. Hoffm. e Euphorbia lycioides Boiss.), uma em áreas de caatinga e brejos de altitude (Croton pulegioides Müll. Arg.) e apenas Cnidoscolus halteris Fdez. Casas e Meinekia neogranatensis (Müll. Arg.) G.L. Webster são encontradas exclusivamente em áreas de brejos de altitude. As demais espécies são exclusivas da Floresta Atlântica e Floresta Amazônica.

Treze espécies são endêmicas da região Nordeste do Brasil (Cnidoscolus byssinus, C. halteris, $C$. infestus, Croton acradenius, C. laceratoglandulosus Caruzo \& Cordeiro, C. lachnocladus, C. pulegioides, C. tenuifolius, C. parodianus Croizat, Dalechampia fernandesii, Discocarpus pedicellatus Fiaschi \& Cordeiro e Jatropha hastifolia).

Quatorze táxons podem ser considerados raros, por apresentarem baixa ocorrência nos acervos locais, coletas restritas a poucas localidades, ou ainda, coletas antigas como: Cnidoscolus byssinus, $C$. halteris, C. hamosus, C. infestus, Croton acradenius, C. lachnocladus, C. parodianus, Discocarpus pedicellatus, Euphorbia lycioides, Flueggea schuechiana (Müll. Arg.) G.L. Webster, Jatropha hastifolia, Meineckia neogranatensis, Podocalyx loranthoides Klotzsch e Tragia friesii Pax \& K. Hoffm.

Acalypha amblyodonta (Müll. Arg.) Müll. Arg., Actinostemon klotzschii (Didr.) Pax, Bernardia celastrinea (Baill.) Müll. Arg., B. micrantha Pax \& K. Hoffm., B. scabra Müll. Arg., B. similis Pax \& K. Hoffm., Dalechampia fernandesii, Flueggea schuechiana, Meineckia neogranatensis, Podocalyx loranthoides e Tragia lessertiana (Baill.) Müll. Arg., conhecidas até então para apenas uma localidade, têm agora a distribuição geográfica ampliada.

Croton sapiifolius Müll. Arg. e Discocarpus pedicellatus são restritas, até o momento, à região do sul da Bahia.

Segundo Melo (2005), Sebastiania Spreng. no nordeste está representado por apenas cinco 
espécies a saber: Sebastiania jacobinensis (Müll. Arg.) Müll. Arg., S. macrocarpa Müll. Arg. ex Müll. Arg., S. trinervia (Müll. Arg.) Müll. Arg., S. brevifolia (Klotzsch ex Müll. Arg.) Müll. Arg. e S. riparia Klotzsch ex Schrad. As demais espécies anteriormente listadas para a região Nordeste foram alocadas em Microstachys A. Juss.

Esser (1994), ao revisar Mabea Aubl. ajustou o número de espécies ocorrentes na região. Este autor informou que Mabea occidentalis Benth., comumente citada em acervos e listagens florísticas para a região Nordeste, ocorre desde o México até oeste do Brasil. Para a região, o autor indicou a ocorrência apenas de Mabea glaziovii Pax \& K. Hoffm., M. piriri Aubl. e M. fistulifera Mart.

Desta maneira, a família Euphorbiaceae para o Nordeste passa a contar com 240 espécies distribuídas em 50 gêneros.

Acalypha amblyodonta (Müll. Arg.) Müll. Arg. in Mart., Fl. Bras. 11(2): 365. 1874 三Acalypha cuspidata Jacq. var. amblyodonta Müll. Arg., Linnaea34:37.1866.Tipo:BRASIL.MinAs GERAIS: Lagoa Santa, s.d., E. Warming s.n. (isótipo P!). Nome popular: pêlo-vermelho.

Material de referência: BRASIL. BAHIA: Feira de Santana, Ipuaçu, 2-XII-2003, J.G. Carvalho Sobrinho 161 (HST); Ilhéus, Arboreto do CEPEC, 4-X-1977, T.S. Santos 3143 (CEPEC, ALCB).

Acalypha, gênero pantropical, reúne cerca de 450 espécies, cujos principais centros de diversidade são o México, a Bolívia e o Peru (Cardiel 1999).

Esta espécie compreende arbustos com ocorrência registrada para o Brasil e norte da Argentina (Govaerts et al. 2000). Ocorre em Floresta Atlântica e em áreas de inselbergues na Bahia. Müller (1873) descreveu quatro variedades para esta espécie (A. amblyodonta var. hispida, A. amblyodonta var. villosa, A. amblyodonta var. gaudichaudii $\mathrm{e}$ A. amblyodonta var. repanda), todas ocorrentes nos estados de Minas Gerais e Rio de Janeiro. Caracteriza-se pelo porte arbustivo, folhas elípticas, membranáceas, de margem serreada a denteada, inflorescência axilar, espiciforme, bissexual, brácteas das flores pistiladas reniformes, denteadas (cerca de 15 dentes), 3 flores pistiladas por bráctea, ovário ovóide, híspido e sementes foveoladas e punctuadas.

Actinostemon klotzschii (Didr.) Pax in Engl., Pflanzenr., IV-147-V: 69. $1912 \equiv$ Dactylostemon klotzschii Didr., Archiv fur Naturgeschichte 7(1):
181. 1841. Tipo: BRASIL. s. loc., s.d., Hagendorf 1347 (holótipo B, possivelmente destruído).

= Actinostemon concepcionis (Chodat \& Hassl.) Hochr., Archiv fur Naturgeschichte 7(1): 181. 1841.

= Actinostemon lasiocarpus (Müll. Arg.) Baill., Adansonia 5: 334.1865.

= Actinostemon communis (Müll. Arg.) Pax in H.G.A.Engler, Pflanzenr., IV-147-V:65. 1912.

$=$ Actinostemon lundianus (Didr.) Pax in H.G.A.Engler, Pflanzenr., IV, 147, V:70 1912 $\equiv$ Dactilostemon lundianus Didr., Vidensk. Meddel. Naturhist. Foren. Kjøbenhavn 1857: 126. 1857.

Material de referência: BRASIL. CEARÁ: Mulungu, Sítio Jardim, 14-II-2003, A.P. Silveira 872 (EAC), Ubajara, Planalto do Ibiapaba, Jaburuna do Sul, 27-I1996, F.S. Araújo s.n (EAC27743); MARANHÃo: São Luís, Reserva Florestal do Sacavem, 19-III-1992, F.H. Muniz 46 (RB).

Actinostemon compreende 13 espécies distribuídas de Cuba até Uruguai e Argentina (Radcliffe-Smith 2001).

Esta espécie é um arbusto a árvore de pequeno porte, registrada para o Brasil, Argentina (Missiones) e Bolívia (Govaerts et al. 2000). Actinostemon klotzschii foi registrada por Jablonski (1969), para os Estados da Bahia, Maranhão e Rio de Janeiro em áreas de solo arenoso, em ambientes de Floresta Atlântica e Restinga. Este trabalho amplia sua distribuição para o Estado do Ceará em vegetação de carrasco. São arvoretas monóicas de folhas espatuladas a lanceoladas, membranáceas, os racemos são axilares a terminais protegidos por brácteas coriáceas; flores estaminadas e pistiladas protegidas por bráctea biglandulosa. Diferencia-se de A. appendiculatus pelo número de flores estaminadas nas címulas (3) e pelas folhas esparsas no ápice dos ramos.

Actinostemon appendiculatus Jabl., Phytologia 18(4): 229. 1969. Tipo: BRASIL. Pernambuco: Nazaré da Mata, s.d., J.C. Moraes 1091 (holótipo US n.v.).

Material de referência: BRASIL. BAHIA: Conceição da Feira, NE da Barragem de Bananeiras, 16-II-1981, A.M. Carvalho 522 (CEPEC).

Espécie registrada para o leste do Brasil (Govaerts et al. 2000) e conhecida até o momento para os Estados da Bahia e Pernambuco. São arbustos com folhas heterófilas, pseudoverticiladas, congestas no ápice dos ramos, bráctea ciliada, flores pistiladas 
crassas, 10-15 flores estaminadas por címula e ovário glabro a esparsamente hirsuto com 6 cornos falcados.

Bernardia celastrinea (Baill.) Müll. Arg. in A.P. D.C., Prodr. 15(2): 921. $1866 \equiv$ Adelia celastrinea Baill., Adansonia 4: 375. 1864. Tipo: BRASIL. Rio DE JANEIRO, 1836, M. Vauthier 37 (síntipo P!). Material de referência: BRASIL. BAHIA: Feira de Santana, Arboreto do CEPEC, 20-III-2001, T.S. Santos et al. 5021 (CEPEC); Ipiaú, Estrada para Jequié, 31-X-1970, T.S. Santos 1252 (CEPEC).

Gênero predominantemente neotropical com 68 espécies, a maior parte delas no Sul do Brasil (Radcliffe-Smith 2001).

Planta arbustiva com cinco variedades descritas por Müller (1873) com base na forma, margem e pilosidade das folhas (B. celastrinea var. capitellata, $B$. celastrinea var. genuina, B. celastrinea var. intermedia, $B$. celastrinea var. obscura e $B$. celastrinea var. serratifolia). As quatro primeiras citadas para a Floresta da Tijuca, no Estado do Rio de Janeiro e $B$. celastrinea var. serratifolia para o Estado de São Paulo. Os espécimes de herbário analisados foram registrados exclusivamente em áreas de Floresta Atlântica.

A espécie pode ser reconhecida pelo hábito arbustivo, ramos dicotômicos, tomentosos, estípulas subuladas, folhas elípticas, cartáceas, serreadas, com ápice agudo, racemos espiciformes minutamente globuliformes.

Bernardia micrantha Pax \& K.Hoffm. in Engl., Pflanzenr. 147, 7 (63): 30. 1914. Tipo: BRASIL. s. loc., s.d., Glaziou 4948 (isótipo P!).

Material de referência: BRASIL. BAHIA: Venceslau Guimarães, 9-V-1969, J.A. Jesus 354 (CEPEC).

Espécie referenciada até então para o Estado do Rio de Janeiro (Govaerts et al. 2000), tem agora sua distribuição ampliada para o Estado da Bahia. Ocorre em área de Floresta Atlântica. São ervas ou subarbustos de folhas elípticas a obovadas, crassas, esparso-serreadas, ápice obtuso a agudo, base aguda, racemos espiciformes longos (ca. $20 \mathrm{~cm}$ compr.), axilares, bractéolas ovais.

Bernardia scabra Müll. Arg. in Mart., Fl. Bras. 11(2): 396. 1874. Tipo: BRASIL. s. loc., s.d., Riedel s.n. (isótipo P!).

Material de referência: BRASIL. BAHIA: Buerarema, rodovia que liga Buerarema à Vila Brasil, km 14, 9-II-1982, A. Carvalho et al. 1149 (CEPEC); Una,
Reserva Biológica do Mico Leão, 30-III-1994, A.M. Amorim 1635 (CEPEC).

Espécie registrada anteriormente apenas para o Estado do Rio de Janeiro (Govaerts et al. 2000). Coletada em Floresta Atlântica na Bahia, em solos argilosos ou arenosos. São plantas arbustivas com cerca de $2 \mathrm{~m}$ alt., folhas subsésseis, membranáceas, flores masculinas em espigas subglobosas, curtamente pedunculadas. Espécie próxima de B. celastrinea, porém diferenciando-se desta pelas estípulas triangular-lanceolatas, ramos não dicotômicos, glabros a discretamente pubescentes.

Bernardia sidoides (Klotzsch) Müll. Arg., Linnaea 34: 177. $1865 \equiv$ Traganthus sidoides Klotzsch, Archiv fur Naturgeschichte 7(1): 188. 1841. Tipo: GUYANA INGLESA [GUIANA], 1836, R.H. Schomburgk 134 (isolectótipo P!).

Material de referência: BRASIL. BAHIA: Iaçu, Fazenda Suibra, Morro do Gado Bravo, 14-III-1985, L.R. Noblick 3701 (CEPEC). CEARÁ: Jaguaretama, Assentamento Brasibel, 20-III-2007, R. Barros 2829 (TJPB). Pernambuco: Mirandiba, Serra do Tigre, 30III-2006, K. Pinheiro 233 (UFP).

Plantas anuais ocorrendo desde o norte da América do Sul até o Brasil (Govaerts et al. 2000). A análise das coleções botânicas indicou a ampla ocorrência desta espécie na região Nordeste, especialmente em áreas de caatinga e cerrado, com solo pedregoso. Diferencia-se das demais espécies, aqui apresentadas, pelo porte herbáceo, ereto, folhas verticiladas (raro alternas), estípulas lineares e flores dispostas em espigas bissexuais, com 1 a 2 flores pistiladas na base das flores masculinas.

Bernardia similis Pax \& K. Hoffm. in Engl., Pflanzenr. IV-147-7: 35. 1914. Tipo: BRASIL. s.loc., s.d., Glaziou 19848 (holótipo B, possivelmente destruído).

Material de referência: BRASIL. BAHIA: Una, Serra do Javi, 3-IV-1986, T.S. Santos et al. 4072 (CEPEC); Unacau, 1988, M. Sobral 5799 (CEPEC).

Espécie anteriormente citada apenas para os Estados de Minas Gerais e Rio de Janeiro (Govaerts et al. 2000). Habita regiões de Floresta Atlântica entre 400 e $700 \mathrm{~m}$ de altitude. Compreende plantas arbustivas com cerca de 1-1,5 m alt., folhas elípticas, cartáceas, irregularmente serreadas, curto-pecioladas, espigas bissexuais, congestas, axilares, de aspecto glomeruliforme. O padrão de ramificação tortuoso é outro caracter que a distingue das demais. 
Cnidoscolus byssinus Fdez. Casas, Fontqueria 55: 82. 2003. Tipo: BRASIL. BAHIA: São Inácio, 6-X-1990, A. Freire-Fierro 1801 et al. (holótipo SPF n.v.). Com distribuição exclusivamente tropical, as 67 espécies de Cnidoscolus (Govaerts et al. 2000) concentram-se principalmente no México e no Nordeste do Brasil (Macbride 1951). No Brasil estão presentes 18 espécies, sendo oito registradas para a região Nordeste (Melo \& Sales 2008).

Cnidosculus byssinus tem ocorrência conhecida, até o momento, para o Estado da Bahia, em área de caatinga com solo pedregoso e a cerca de $500 \mathrm{~m}$ de altitude. Caracteres que auxiliam seu reconhecimento são as flores estaminadas obpiriformes, 6-verticiladas, além da ocorrência de 29 a 30 estames e 4 a 5 estaminódios.

Rara pela baixa representatividade nos acervos locais e coletas restritas a poucas localidades.

Cnidoscolus halteris Fdez. Casas, Fontqueria 55: 34. 2001. Tipo BRASIL. Pernambuco: Brejo da Madre de Deus, Fazenda Bituri, 26-V-1995, F. Villarouco et al. 85 (holótipo NY!).

Material de referência: BRASIL. Pernambuco: Brejo da Madre de Deus, Fazenda Bituri, 9-II-1993, S.I. Silva 52 (PEUFR); Santa Cruz de Capibaribe, Jataúba, km 16, 8-II-1993, s.col. 49 (PEUFR39607).

Ocorre nos Estados da Bahia e Pernambuco (Casas 2001a). São subarbustos a arbustos com altura variando de 0,8-1,5 m, látex transparente, pecíolo com 6-12 cm compr., hirto-viloso, bráctea vilosa, cálice das flores estaminadas viloso e estames-10, livres. Habita áreas com solos argilosos e ambientes pedregosos e com altitudes variando entre $400 \mathrm{e}$ $1.000 \mathrm{~m}$, sendo restrita a poucas localidades.

Cnidoscolus hamosus Pohl, Pl. Bras. icon. descr. 1: 57. 1827. Tipo: BRASIL. Piauí: s. loc., 1839, G. Gardner 2301 (isótipo K!).

Distribuição restrita, até o momento, ao Estado do Piauí (Casas 2001b). São árvores de 4-5 m de alt. com folhas minutamente crenadas, 18 a 20 estames férteis, dispostos em 4 verticilos e as flores com mais de $2 \mathrm{~cm}$ compr. Ocorre em vegetação de Caatinga com afloramentos rochosos graníticos. Considerada aqui como rara devido à baixa amostragem nas coleções examinadas e coletas restritas a poucas localidades.

Cnidoscolus infestus Pax \& K. Hoffm. in Engl., Pflanzenr. IV, 147, 16 (85): 193. 1924. Tipo: BRASIL. PARAíba: Serra da Borborema,
Lutzelburg 12428 (lectótipo M!, aqui designado).

= Cnidoscolus piranii Fdez. Casas \& Pizarro, Fontqueria 55(17): 89. 2003.

Nome popular: cansanção.

Material de referência: BRASIL. PARAíBA: Campina Grande, São José da Mata, Fazenda Pedro da Costa Agra, estrada para Soledade, 25-VI-1993, M.F. Agra 2091 (JPB).

Espécie endêmica do Brasil (Bahia, Ceará, Minas Gerais, Paraíba e Pernambuco), ocorrendo em vegetação de Caatinga de acordo com Casas \& Domínguez (2005). Caracteriza-se pelas folhas longo-pecioladas, profundamente 5-partidas, lobos oblongo-lanceolados, agudos, dentados, dentes glandulosos no ápice, inflorescências axilares ou terminais, pedúnculo e disco glandular das flores estaminadas viloso. Considerada aqui como rara devido a baixa amostragem nas coleções examinadas e coletas restritas a poucas localidades.

Croton acradenius Pax \& K. Hoffm., Repert. Spec. Nov. Regni Veg. 41: 224. 1937. Tipo: BRASIL. CEARÁ: Cedro, 31-V-1933, P. Luetzelburg 26482 (síntipo M!).

Figura 1A-E

Material de referência: BRASIL. PIAUí: Rodovia PI 030 entre Teresina e Palmeiras, 29-IX-1986, A. Fernandes \& E. Nunes s.n. (EAC8794, UFP50410).

Considerado o segundo maior gênero de Euphorbiaceae s.s., com cerca de 1.200 a 1.300 espécies (Berry et al. 2005). A maioria das espécies está distribuída na América do Sul e Antilhas, algumas na América do Norte (Webster 1994), África e Madagascar (Webster 1993). Suas espécies incluem ervas, arbustos, subarbustos e ocasionalmente lianas (Secco 2008), destacando-se como um dos gêneros mais representativos na região Nordeste do Brasil com número estimado em 70 espécies (CarneiroTorres 2009).

Croton acradenius com ocorrência anteriormente restrita ao Estado do Ceará (Govaerts et al. 2000), passa agora a ter distribuição ampliada para o Estado do Piauí. Habita áreas de caatinga arbustiva densa e de cerrado. Reconhecida pelo hábito arbustivo, com ramos e folhas ferruginosos com tricomas estrelados, folhas estreito-elípticas, racemos terminais, congestos, além de sépalas, pétalas e ovário com tricomas estrelados longo-estipitados. A análise do typus revelou estreita semelhança desta com $C$. pedicellatus Kunth, sendo ambas, possivelmente, uma só espécie. Considerada aqui como rara devido à 


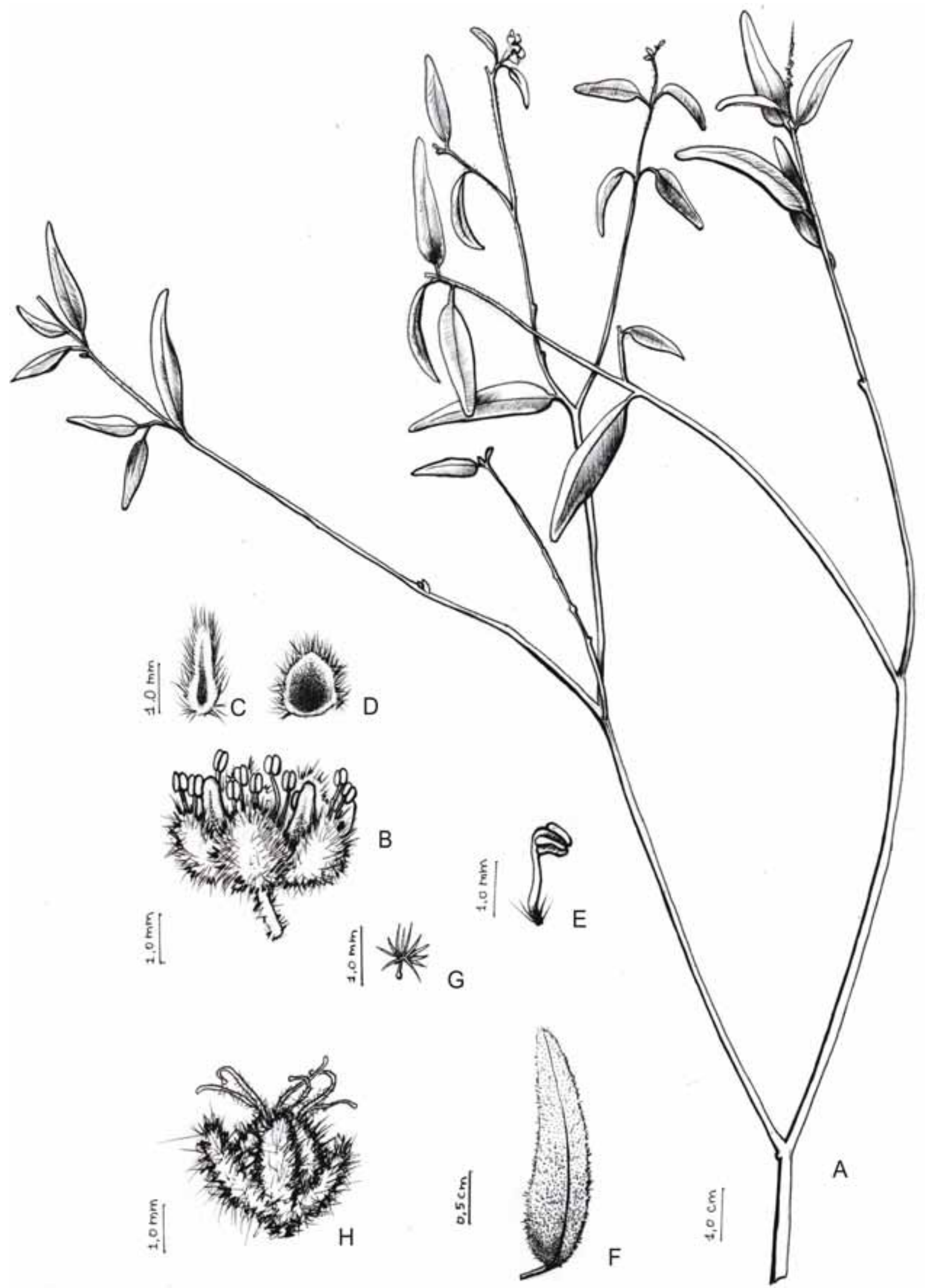

Figura 1. Croton acradenius. A. Ramo com flores. B. Flor estaminada. C. Pétala da flor estaminada. D. Sépala da flor estaminada. E. Estame. G. Tricoma estrelado-estipitado (A. Fernandes \& E. Nunes EAC8794).

Figure 1. Croton acradenius. A. Branch with flowers. B. Staminate flower. C. Staminate flower petal. C. Staminate flower sepal. E. Stamen. G. stipitate-stellate trichomes. (A. Fernandes \& E. Nunes EAC8794). 
baixa amostragem nas coleções examinadas e coletas restritas a poucas localidades.

Croton laceratoglandulosus Caruzo \& Cordeiro, Bot. J. Lin. Soc. 158: 493. 2008. Tipo: BRASIL. BAHIA: Malhada, 1-IV-2001, fl., fr., J.G. Jardim et al. 3372 (holótipo CEPEC! isótipo HUEFS!).

Material de referência: BRASIL. CEARÁ: Reserva Biológica Serra de Aiuaba, 14-III-2005, J.R. Lemos 258 (UFP). Pernambuco: Mirandiba, Fazenda Baixa Grande, 19-IV-2007, J.R. Maciel et al. 447 (UFP). Piauí: Serra da Capivara, 1979, L. Emperaire 623(IPA).

Espécie recentemente descrita com distribuição disjunta entre o semiárido brasileiro (BA, CE MG, PI) e sudeste da Bolívia (Cordeiro et al. 2008), sendo comum em áreas de florestas secas e caatingas com altitudes de 300-820 m. São plantas arbustivas com 0,7-2 m alt., folhas cordadas a largo-elípticas, látex incolor, ramos pilosos, tricomas denso-estrelados, estípulas longas (10-15 mm compr.) e profundamente lacerato-glandulosas, brácteas lacerato-glandulosas e estiletes multífidos.

Croton lachnocladus Müll. Arg. in Mart., Fl. Bras. 11(2): 258. 1873. Tipo: BRASIL. BAHIA: Vila Nova da Rainha, s.d., Martius 2269 (holótipo $\mathrm{M}$ !, isótipo $\mathrm{K}$ !).

Espécie registrada apenas para o Estado da Bahia, em área de caatinga. De acordo com Müller (1873) são arbustos com ramos di a tricotômicos, cilíndricos, glabros, cinéreos e com folhas lanceoladas a ovais. Ainda segundo esse autor, os racemos se assemelham aos de Croton rhamnifolius H.B.K. [= C. heliotropiifolius Kunth] exceto por esta apresentar base do eixo floral com numerosas e adensadas flores pistiladas. Análise do typus confirmou a estreita semelhança com $C$. heliotropiifolius. Considerada aqui como rara devido a baixa amostragem nas coleções examinadas e coletas restritas a poucas localidades.

Croton parodianus Croizat, Darwiniana 6: 452. 1944. Tipo: BRASIL. Maranhão: São Luiz, IIIV-1939, R.L. Fróes 11775 (holótipo K!).

Nome popular: velame.

Espécie com distribuição restrita à Venezuela e Guiana (Carneiro-Torres 2009). Para o Brasil é conhecida até o momento para o Estado do Maranhão (Govaerts et al. 2000). São arbustos habitando florestas com solo pedregoso. Pode ser reconhecida pelas folhas longo-pecioladas, largo-cordadas, dentadas, com nectários extraflorais estipitadocaliciformes entre os dentes e no ápice do pecíolo, apresenta ainda racemos longos (cerca de $25 \mathrm{~cm}$ compr.), bractéolas com glândulas obpiriformes e as sépalas pistiladas, profundamente recortadas e afastadas uma das outras e flores e folhas densoestreladas. Considerada aqui como rara devido à baixa amostragem nas coleções examinadas e distribuição restrita.

Croton pulegioides Müll. Arg. in Mart., Fl. Bras. 11(2): 259. 1873. Tipo: BRASIL. BAHIA: Jacobina, s.d., Blanchet 3786 (isótipo K!). Nomes populares: velandinho, barba-de-bode. Material de referência: BRASIL. Pernambuco: Bezerros, Parque Ecológico Serra Negra de Bezerros, 12-IV-1995, M.F. Sales 572 (PEUFR). Piauí: São Raimundo Nonato, Clemente, 11-IV1979, M.R. Del'Arco s.n. (TEPB968). Sergipe: Nossa Senhora da Glória, Fazenda Olho D’Água, 6-V-1986, G. Viana 1458 (ASE).

Plantas arbustivas e endêmicas da região Nordeste do Brasil (Govaerts et al. 2000). Comuns em brejos de altitude e caatinga. Apresentam 1-2 $\mathrm{m}$ alt., ramos dicotômicos, aroma amentolado e folhas membranáceas com 2-4 glândulas (nectários extraflorais) cilíndricas, estipitadopateliformes ou caliciformes na base da lâmina. Morfologicamente similar a $C$. pulegiodorus Baill. e C. tetradenius Baill., sendo necessários mais estudos para melhor delimitação entre os táxons.

Croton sapiifolius Müll. Arg., Linnaea 34: 137. 1865. Tipo: BRASIL. s.loc., s.d., Sello 557 (holótipo B, possivelmente destruído).

Material de referência: BRASIL. BAHIA: Una, Reserva Biológica do Mico-Leão, Picada da Bandeira, 23-III-1997, A.L. Santos 601 (CEPEC).

Espécie com ocorrência conhecida apenas para o Estado da Bahia em Floresta Atlântica (Carneiro-Torres 2009). Diferencia-se das espécies morfologicamente mais relacionadas pelo hábito arbustivo com ca. $3 \mathrm{~m}$ alt., folhas largoovais, ca. $20 \mathrm{~cm}$ compr., longo-pecioladas, largoovais a obovadas, cartáceas, glabras, margem inteira, nervuras impressas e um par de nectários extraflorais, sésseis e depresso-globosos, no ápice do pecíolo. Considerada aqui como rara devido a baixa amostragem nas coleções examinadas e coletas restritas a poucas localidades. 
Croton tenuifolius Pax \& K. Hoffm., Repert. Spec. Nov. Regni Veg. 41: 225. 1937. Tipo: BRASIL. CeArá: Granjeiros, Tabuleiros, 20-V-1934, $P$. Luetzelburg 2691 (isótipo M!).

Material de referência: BRASIL. Piauí: Oeiras, Exu, 14-IV-2007, F.C.S. Oliveira et al. 10 (TEPB).

Subarbustos com distribuição restrita aos Estados do Piauí e Ceará (Govaerts et al.2000), principalmente em áreas de florestas úmidas (Carneiro-Torres 2009). Caracteriza-se por apresentar ramos di-tricotômicos, quadrangulares, um par de estípulas lineares a setáceas, folhas elípticas, membranáceas, com um par de nectários extraflorais estipitado-pateliformes. A face adaxial das folhas apresenta tricomas simples e estrelados. Outros caracteres que auxiliam seu reconhecimento são as sépalas das flores pistiladas linear-lanceoladas e a ocorrência de sementes foveoladas. A similaridade com Croton betaceus Baill. sugere que estudos são necessários para revelar a correta identidade de ambos. Esta última é comumente citada para áreas de carrasco e cerrado no Estado do Piauí (Araújo et al. 1998, Santos Filho 2000).

Dalechampia fernandesii G.L. Webster, Brittonia 41: 1. 1989. Tipo: BRASIL. CEARÁ: Tianguá, Chapada da Ibiapaba, 1-XI-1986, G. Webster et al. 25598 (holótipo EAC!).

Material de referência: BRASIL. CEARÁ: Aiuaba, Estação Ecológica de Aiuaba, 9-IX-2004, J.R. Lemos \& P. Matias 264 (EAC, IPA, UFP).

Gênero pantropical, mas predominantemente neotropical com 110 espécies, sendo 90 delas americanas (Radcliffe-Smith 2001). Dalechampia fernandesii é uma liana urticante com distribuição indicada por Webster (1989) para o oeste do Ceará em áreas montanhosas. $\mathrm{O}$ autor afirma ser esta espécie próxima de $D$. tiliifolia Lam., porém diferenciandose desta pelas folhas trilobadas, presença de 18-22 estames e disco estigmático largo, e de $D$. affinis Müll. Arg. pela presença das bractéolas trilobadas, estilete curto e disco estigmático largo. A ocorrência na região é confirmada para Floresta Pluvial com 770 $\mathrm{m}$ alt. e em área de Caatinga entre 400 e $600 \mathrm{~m}$ alt.

Discocarpus pedicellatus Fiaschi \& Cordeiro, Brittonia 57: 248. 2005. Tipo: BRASIL. BAHIA: Jussari, Rod. Jussari/Palmira, acesso para sede da Fazenda Teimoso, RPPN Serra do Teimoso, 11-X-2003, J.L. Paixão et al. 1672 (holótipo CEPEC!, isótipo HUEFS!).
Gênero incluído em Phyllanthaceae por Wurdack et al. (2005). Abriga apenas três espécies comuns em áreas ripárias na região Amazônica (Radcliffe-Smith 2001). Discocarpus pedicellatus é representada por árvores dióicas, com cerca de 6-15 m alt., endêmica das Florestas Semidecíduas da região sul da Bahia (Fiaschi \& Cordeiro 2005). Apresenta folhas concolores, flores pistiladas com pedicelos de 8-10 $\mathrm{mm}$ compr. e estiletes frequentemente unidos em coluna, com 2,5-3 mm compr. e frutos imaturos com indumento ferrugíneo-estriado. Segundo os autores, diferencia-se de D. brasiliensis Klotzsch ex Müll. Arg. (= D. essequeboensis Klotzsch), pelos botões florais terminais, glabros a minutamente hirsutos, ovário muricado a reticulado e flores estaminadas com 2(3) pistilóides. Esta última espécie, por sua vez, ocorre desde o norte da América do Sul até o Brasil, com registros também para a Bahia. Considerada aqui como rara devido à baixa amostragem nas coleções examinadas e limitada área de ocorrência.

Euphorbia lycioides Boiss., Cent. Euphorb.: 29. 1860. Tipo: BRASIL. PiAúi: s. loc., s.d., Gardner 1829 (isótipo $\mathrm{K} !$ ).

Figura $2 \mathrm{~A}-\mathrm{G}$

Nome popular: carqueja.

Material de referência: BRASIL. BAHIA: Palmeiras, Caminho para Serra Preta, 28-III-2003, E. Melo 3640 (HUEFS). Piauí: Parque Nacional Serra da Capivara, 27-IV-2007, M.F.A. Lucena 1725 (UFP).

Euphorbia L. apresenta distribuição subcosmopolita sendo considerado o maior gênero de Euphorbiaceae s.s. com cerca de 2.000 espécies (Radcliffe-Smith 2001). Steinmann \& Porter (2002) e Steinmann (2003) alteraram a circunscrição do gênero com a inclusão de Chamaesyce Gray, Pedilanthus Neck. ex Poit. e Poisenttia Graham.

Euphorbia lycioides é restrita, até o momento, aos Estados do Piauí e Bahia e coletada sobre afloramentos rochosos em área de caatinga. São arbustos latescentes, com cerca de $1 \mathrm{~m}$ alt., com caule lignificado e ramos acinzentados, tomentosos, canaliculados, tortuosos; as folhas são elípticas a obovadas, sésseis, suculentas, conduplicadas, com ápice acuminado a emarginado, glabras. Apresenta um par de estípulas ovóides, glandulígeras. $\mathrm{O}$ invólucro caliciforme do ciátio é campanulado, alvo, glabro externamente e viloso internamente com margem denteada e revoluta, o ovário é glabro. A espécie apresenta forte potencial ornamental e é considerada aqui como 


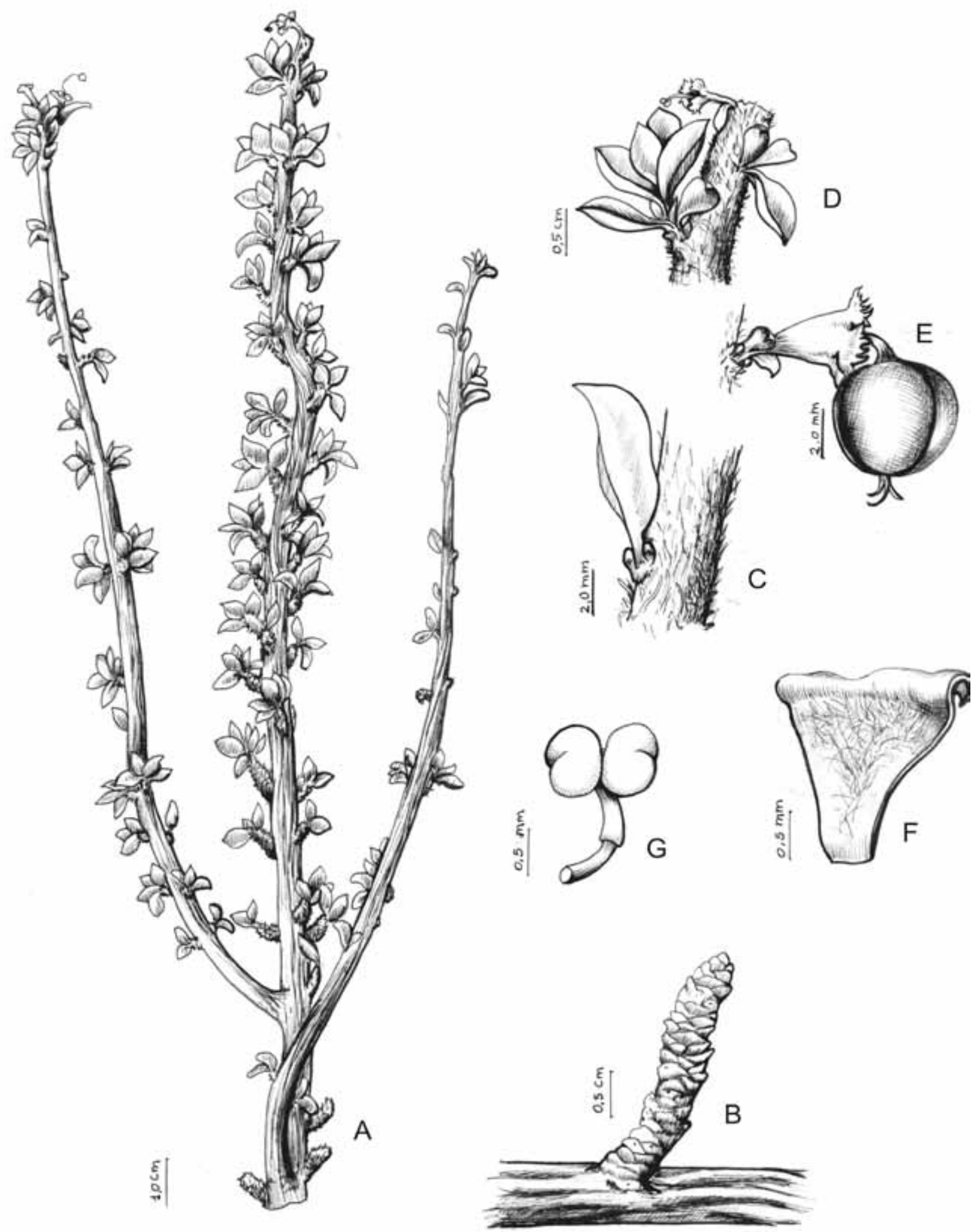

Figura 2. Euphorbia lycioides. A. Ramo com flor e fruto. B. Aspecto do ramo mais basal envelhecido. C. Estípulas. D. Ciátio. E. Fruto. F. Detalhe da parte interna do invólucro caliciforme. G. Estame. (M.F.A. Lucena 1725).

Figure 2. Euphorbia lycioides. A. Branch with flowers and fruit. B. Aspect of the most basal branch aged. C. Stipule. D. Cyathium. E. Fruit. F. Detail of inside of caliciform-involucre. G. Stamen. (M.F.A. Lucena 1725). 
rara devido à baixa amostragem nas coleções examinadas e coletas restritas a poucas localidades.

Flueggea schuechiana (Müll. Arg.) G.L. Webster, Allertonia 3: 277. 1984 ESecurinega schuechiana Müll. Arg. Tipo: BRASIL. s. loc., s.d., Schuec s.n. (holótipo W n.v.).

Figura 3A-E

Material de referência: BRASIL. BAHIA: Feira de Santana, Fazenda Monte Verde, 21-VII-1987, L.P. Queiroz 1744 (HUEFS, IPA); Fazenda Roça da Serra, Serra de São José, 30-III-1984, L.R. Noblick 3123 (PEUFR).

Flueggea Willd., incluído em Phyllantaceae (Kathariachchi et al. 2005), é representado por arbustos ou árvores dióicos ou raramente monóicos, amplamente distribuídos nos trópicos e Eurásia temperada, mas com distribuição relictual disjuncta (Radcliffe-Smith 2001). Esser (2003) indicou a distribuição do gênero apenas para os Paleotrópicos e Eurásia. Registrada por Webster (1984) apenas para Pernambuco, através de uma coleta realizada em 1954, tem agora a distribuição ampliada para o Estado da Bahia. Espécie frequentemente confundida com Savia dyctiocarpa Müll. Arg., (Webster 1984), porém diferencia-se desta, pelas pétalas das flores pistiladas maiores, flores estaminadas pediceladas e ovário pubescente. Considerando o longo período de ausência de coletas da mesma, os escassos exemplares nos acervos e sua restrita ocorrência, pode-se tratá-la como espécie rara.

Jatropha hastifolia Fdez. Casas, Fontqueria 55: 109. 2003. Tipo: BRASIL. BAHIA: Remanso, Saída de Remanso para Pilão Arcado, 28-II-2000, L. Passos 388 et al. (holótipo CEN, isótipo ALCB!).

Casas (2003) indicou esta espécie como rara e possivelmente endêmica da região do vale do rio São Francisco, ao norte do Estado da Bahia. Segundo o autor, compreende arbustos com $2 \mathrm{~m}$ de altura, caule viloso, folhas hastadas, membranáceas e vilosas, flores estaminadas com disco glandular viloso, pétalas avermelhadas e sementes com carúncula esbranquiçada.

Meineckia neogranatensis (Müll. Arg.) G.L. Webster subsp. hilariana (Baill.) G.L. Webster, Acta Bot. Neerl. 14: 349. $1965 \equiv$ Flueggea hilariana Baill., Adansonia 5: 346. $1865 \equiv$ Securinega hilariana (Baill.) Müll. Arg. in A.P. D.C., Prodr. 15(2): 1273. $1866 \equiv$ Acidoton hilarianus (Müll.
Arg.) Kuntze, Revis. Gen. Pl. 2: 592. 1891. Tipo:

BRASIL. Minas GERAIS: s.d., A. St-Hilaire 1488 (holótipo P!).

Material de referência: BRASIL. CEARÁ: Serra de Pacatuba, Sítio Pitaguari, 17-II-1968, A. Lima 68-5290 (IPA). Pernambuco: Triunfo, Sítio Olho D'Água, 4-XI-1992, E. Ferraz 205 (IPA, PEUFR).

Meineckia Baill., atualmente inserido em Phyllanthaceae (Wurdack et al. 2005), é constituído por 20 espécies e tem distribuição disjunta entre o Velho e o Novo Mundo; ocorrendo desde o México até Colômbia e Brasil e da África Central a Madagascar, Oriente Médio e sudeste Asiático (Radcliffe-Smith 2001). No Brasil, esta espécie foi registrada por Müller (1873) para o Estado de Minas Gerais como Securinega hilariana (Baill.) Müll. Arg. São árvores de pequeno porte (cerca de $4 \mathrm{~m}$ alt.), ocorrendo em brejos de altitude dos Estados do Ceará e Pernambuco. Considerada aqui como rara devido a baixa amostragem nas coleções examinadas e limitada área de ocorrência.

Podocalyx loranthoides Klotzsch, Arch. Naturgesch. 7: 202.1841 E Richeria loranthoides (Klotzsch) Müll. Arg. in A.P. D. C., Prodr. 15(2): 469.1866. Tipo: BRASIL. Amazonas: Rio Negro, s.d., Schomburgk 978 (holótipo B, possivelmente destruído, n.v.).

Material de referência: BRASIL. AlagoAs: Cacimbinhas, 12-V-1982, M. Staviski 914 (MAC).

Gênero monotípico e, segundo Wurdack et al. (2005), inserido em Picrodendraceae. Espécie conhecida para a região amazônica, Colômbia, Venezuela, Peru e Brasil (Webster \& Berry 1999, Radcliffe-Smith 2001). A ocorrência em área de Caatinga no Estado de Alagoas é o primeiro registro extra-amazônico da espécie. Habita áreas com solos bem drenados de caatinga arbórea. São arbustos ou arvoretas, dióicas, com folhas elípticas, membranáceas, com cerca de $15 \mathrm{~cm}$ compr., margem inteira, base atenuada, glabras, estípulas caducas, inflorescência axilar com flores estaminadas e pistiladas dispostas em pseudoespigas solitárias, flores estaminadas e pistiladas monoclamídeas e com pedicelo tomentoso-ferrugíneo. Único registro conhecido para a região Nordeste do Brasil, sendo por isto considerada rara.

Philyra brasiliensis Klotzsch, Arch. Naturgesch. 1: 199. $1841 \equiv$ Argyrothamnia brasiliensis (Klotzsch) Müll. Arg., Linnaea 34: 144. 1865. 

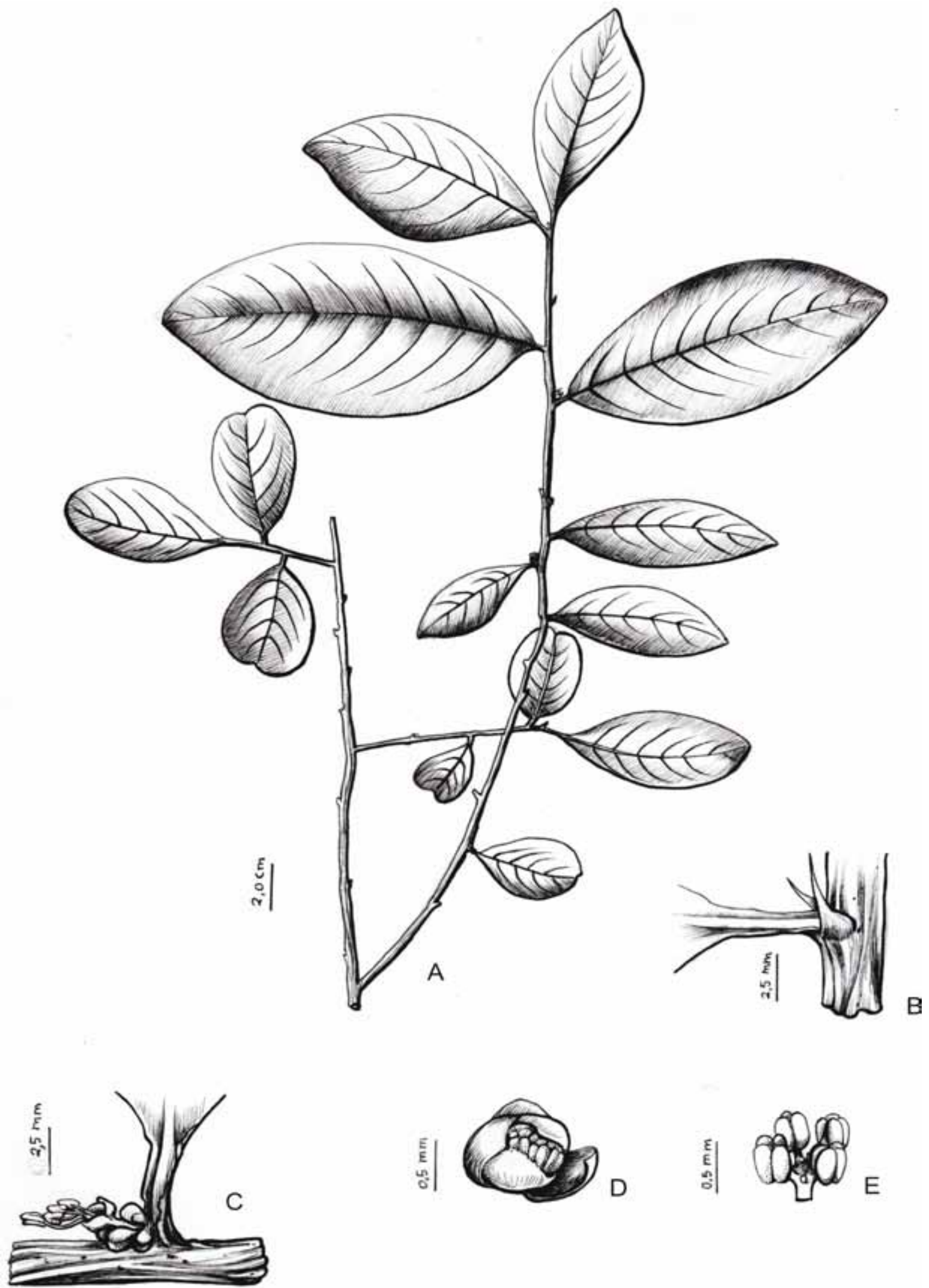

Figuras 3. Flueggea schuechiana. A. Ramo florido. B. Estípulas. C. Botões florais estaminados. D. Detalhe do botão floral masculino. E. Estames. (L.P. Queiroz 1744).

Figure 3. Flueggea schuechiana. A. Flowering branch. B. Stipule. C. Staminate flowers buds. D. Detail of the male flowers buds. E. Stamens. (L.P. Queiroz 1744). 
Tipo: BRASIL. Rio DE JANEIRO: s.d., Sellow s.n. (holótipo B, possivelmente destruído, isótipo MBG!).

Material de referência: BRASIL. AlagOAs: União dos Palmares, Serra Pelada, 11-III-1982, M. Staviski \& R. Lyra-Lemos 904 (MAC). BAHIA: Ilhéus, Arboreto do CEPEC, 20-IX-2002, T.S. Santos 345 (CEPEC).

Gênero monotípico registrado para o Paraguai e o Sul do Brasil (Radcliffe-Smith 2001). Espécie com distribuição indicada por Govaerts et al. (2000), para o Brasil até o nordeste da Argentina, sendo aqui registrada para os Estados de Alagoas e Bahia em Florestas Estacionais. São arbustos dióicos com ramos espinescentes e folhas alternas, curto-pecioladas, cartáceas e com tricomas simples. As flores são dispostas em inflorescências axilares, racemosas, sendo as estaminadas mais adensadas que as pistiladas, estames 15 , dispostos em 3 verticilos. Pode ser confundida com algumas espécies do gênero Ditaxis Vahl ex A. Juss., mas diferenciase pela ausência de tricomas malpighiáceos e pela presença dos ramos espinescentes. Considerada aqui como rara devido à baixa amostragem nas coleções examinadas e limitada área de ocorrência.

Savia dictyocarpa Müll. Arg. in Mart. Fl. Bras. 11(2): 704. 1873. Tipo: BRASIL. Rio DE JANEIRo: Glaziou 5986 (holótipo G n.v.).

= Securinega guaraiuva Kuhlmann, Arq. Inst. Biol. Veg. Rio de Jan. 1: 241. 1935.

Material de referência: BRASIL. BAHIA: Anguera, Morro da Fazenda Retiro, 29-IV-1999, F. França 2694 (HUEFS). Pernambuco: Ilha de Fernando de Noronha, 22-X-2004, A.M. Miranda 4508 (HST). Rio GRande do Norte: Jucurutu, RPPN Stoessel de Britto, 7-IX-2007, A.A. Roque 300 (UFP, UFRN). SERGIPE: Porto da Folha, Fazenda São Pedro, 14-VI2007, M.F.A. Lucena et al. 1831 (ASE, UFP).

Incluído em Phyllanthaceae (Wurdack et al. 2005) o gênero era constituído, até recentemente, por 25 espécies da América Tropical, Leste e Sul da África e Madagascar (Radcliffe-Smith 2001). Hoffmann et al. (2006), Hoffmann \& McPherson (2007) e Hoffmann (2008) transferiram as 13 espécies africanas para Blotia Lendri e Petalodiscus Baill., ficando agora o gênero restrito a $S$. dictyocarpa e $S$. sessiliflora (Sw.) Willd. De acordo com Webster \& Berry (1999), S. dictyocarpa é um táxon comum em florestas semidecíduas do Panamá, Venezuela, Cuba, México, Porto Rico e, no Brasil, representada por arbustos a arvoretas, da Floresta Atlântica do Sudeste e Sul do país (Müller 1873, Govaerts et al. 2000, Pax \& Hoffman 1922) sendo este, o primeiro registro para áreas de caatinga.

Pode ser reconhecida pelo hábito arbóreo, pecíolo rugoso e piloso, pétalas nas flores estaminadas ausentes, cinco estames, fruto oblongo, rugoso e sementes aplanadas, castanhas, brilhantes e foveoladas. Diferencia-se de S. sessiliflora por esta ser restrita à região das Antilhas (Pax \& Hoffman 1922, Webster \& Berry 1999) e pela ausência de pétalas nas flores estaminadas.

Tragia friesii Pax \& K. Hoffm. in Engl., Pflanzenr., IV, 147, XVII: 186. 1924. Tipo: BOLÍVIA. CHACO: Tatarenda, 17-III-1902, Fries 1383 (holótipo S n.v.).

Material de Referência: BRASIL. BAHIA: Milagres, Morro Pé de Serra, 12-III-2001, F. França 2169 (ALCB).

Tragia é um gênero pantropical com cerca de 150 espécies, distribuídas principalmente na América Tropical e África, com apenas três espécies ocorrentes na Ásia Tropical (Radcliffe-Smith 2001). Tragia friesii é uma trepadeira urticante com distribuição indicada para a Bolívia e Argentina (Govaerts et al. 2000), sendo aqui registrada a primeira ocorrência para o Brasil em Floresta Atlântica no Estado da Bahia entre 350 e $400 \mathrm{~m}$ de altitude. Pode ser reconhecida pelas folhas cordadas, longo-pecioladas e racemos bissexuais com menos de $10 \mathrm{~cm}$ compr.

Tragia lessertiana (Baill.) Müll. Arg., Linnaea 34: 178. $1865 \equiv$ Bia lessertiana Baill., Étude Euphorb.: 502. 1858. Tipo: BRASIL. s.loc., s.d., s.col. (holótipo G!).

Material de Referência: BRASIL. BAHIA: Feira de Santana, BA-052, 22-XI-1986, L.P. Queiroz et al. 1380 (HUEFS, MAC, TEPB). Pernambuco: Igarassu, Usina São José, 10-I-2008, A. Alves-Araújo et al. 799 (UFP).

Esta espécie é indicada para as Guianas e Brasil (Amapá e Maranhão) de acordo com Govaerts et al. (2000), ampliando-se aqui os registros para os Estados da Bahia e Pernambuco. São plantas escandentes e urticantes de folhas membranáceas, comuns em Floresta Atlântica, em área de encosta (360-400 m de altitude). Diferencia-se de $T$. friesii pelas folhas oblanceoladas, pelos racemos aos pares, unissexuados, com ca. de $15 \mathrm{~cm}$ compr., bractéolas lanceoladas, subuladas, lacínios do cálice feminino linear-lanceolados, estames 15-20, ovário híspido e estiletes longos, papilosos. 
Os dados aqui apresentados indicam a grande importância das coleções botânicas como banco de dados sobre a biodiversidade e a necessidade da realização de levantamentos criteriosos nos herbários da região. Os resultados apontam para novos dados sobre Euphorbiaceae, ajustando e atualizando informações sobre o checklist da família na região Nordeste.

\section{Agradecimentos}

Os autores agradecem aos curadores, técnicos e funcionários dos herbários visitados; ao Dr. Hans-Joachim Esser, por disponibilizar bibliografia especializada e identificação de alguns táxons; ao Conselho Nacional de Desenvolvimento Científico e Tecnológico $(\mathrm{CNPq})$ e à Fundação $\mathrm{O}$ Boticário de Proteção à Natureza pelo apoio financeiro (Processo 0688-2005); à Regina Carvalho pelas ilustrações.

\section{Literatura citada}

Alcoforado Filho, F.G., Sampaio, E.V.S.B. \& Rodal, M.J.N. 2003. Florística e fitossociologia de um remanescente de vegetação caducifólia espinhosa em Caruaru, Pernambuco. Acta Botanica Brasilica 17: 287-303.

Andrade, K.V.S.A., Rodal, M.J.N., Lucena, M.F.A. \& Gomes, A.P.S. 2004. Composição florística de um trecho do Parque Nacional do Catimbau, Buíque, Pernambuco-Brasil. Hoehnea 31: 337-348.

APG. 2003. An update of the Angiosperm Phylogenetic Group classification for the orders and families of flowering plants: APGII. Botanical Journal of the Linnean Society 141: 399-436.

Araújo, E.L., Sampaio, E.V.S.B. \& Rodal, M.J.N. 1995. Composição florística e fitossociológica de três áreas de caatinga. Revista Brasileira de Biologia 55: 595-607.

Araújo, F.S., Sampaio, E.V.S.B., Figueiredo, M.A., Rodal, M.J.N. \& Fernandes, A.G. 1998. Composição florística da vegetação de carrasco, Novo Oriente, CE. Revista Brasileira de Botanica 21: 105-116.

Barroso, G.M., Guimarães, E.F. \& Ichaso, C.L.F. 1991. Sistemática de angiospermas do Brasil. v. 2. Universidade de São Paulo, São Paulo.

Berry, P.E., Hipp, A.L., Wurdack, K.J., Van Ee, B. \& Riina, R. 2005. Molecular phylogenetics of the giant genus Croton and tribe Crotoneae (Euphorbiaceae sensu stricto) using ITS and trnL-trnF DNA sequence data. American Journal of Botany 92: 1520-1534.

Cardiel, J.M. 1999. Contribuciones a la flora de Venezuela: Revisión del género Acalypha L. (Euphorbiaceae). Acta Botanica Venezuelica 22: 255-324.

Carneiro-Torres, D.S. 2009. Diversidade de Croton no Bioma Caatinga. Tese de Doutorado, Universidade Estadual de Feira de Santana, Feira de Santana.

Casas, F.J.F. 2001a. Cnidoscolorum notulae (Euphorbiaceæ), 1-5. Fontqueria 55: 33-38.

Casas, F.J.F. 2001b. Synopsis of Cnidoscolus series Cnidoscolus (Euphorbiaceae), with description of a new species. Fontqueria 55: 39-46.

Casas, F.J.F. 2003. Una especie nueva, de Brasil: Jatropha hastifolia. Fontqueria 55: 109-112.

Casas, F.J.F. \& Dominguez, J.M.P. 2005. Cnidoscolorum notulae (Euphorbiaceae): $C$. infestus Pax \& K. Hoffm. Adumbrationes ad Summae Editionem 13: 1-28.

Chase, M.W., Zmarzty, S., Lledó, M.D., Wurdack, K.J., Swensen, S.M. \& Fay, M.F. 2002. When in doubt, put in Flacourtiaceae: a molecular phylogenetic analysis based on plastid $r b c L$ DNA sequences. Kew Bulletin 57: 141-181.

Cordeiro, I. \& Carneiro-Torres, D.S. 2006. Euphorbiaceae. In: M.R.V. Barbosa, C. Sothers, S. Mayo, C.F.L. Gamarra \& A.C. Mesquita. (orgs.). Checklist das plantas do Nordeste brasileiro v.1: Angiospermas e Gymnospermas. Ministério da Ciência e Tecnologia, Brasília, pp.71-74.

Cordeiro, I., Berry, P.E., Caruzo, M.B.R. \& Van Ee, B. 2008. Croton laceratoglandulosus Euphorbiaceae s.s., a new glandular-stipulate species from Brazil and Bolivia, and its systematic position based on molecular analysis. Botanical Journal of the Linnean Society 158: 493-498.

Esser, H-J. 1994. Systematische Untersuchungen an den Hippomaneae Adr. Jussieu ex Bartling (Euphorbiaceae), insbesondere den Mabeinae Pax \& K.Hoffm. Doctoral dissertation, University of Hamburg, Hamburg.

Esser, H-J. 2003. Fruit characters in Malesian Euphorbiaceae. Telopea 10: 169-177.

Fiaschi, P. \& Cordeiro, I. 2005. Discocarpus pedicellatus, a new species of Phyllanthaceae 
(Euphorbiaceae s.1.) from southern Bahia, Brazil. Brittonia 57: 248-251.

Govaerts, R., Frodin, D.G. \& Radcliffe-Smith, A. 2000. World checklist and bibliography of Euphorbiaceae (and Pandaceae) v.1-4. Royal Botanical Gardens, Kew.

Hoffmann, P. 2008. Revision of Heterosavia, stat. nov., with notes on Gonatogyne and Savia (Phyllanthaceae). Brittonia 60: 136-166.

Hoffmann, P. \& McPherson, G. 2007. Revision of Wielandia including Blotia and Petalodiscus (Phyllantaceae, Euphorbiaceae s.l.). Annals of the Missouri Botanical Garden 94: 519-553.

Hoffmann, P., Kathriarachchi, H. \& Wurdack, K.J. 2006. A phylogenetic classification of Phyllanthaceae (Malpighiales; Euphorbiaceae sensu lato). Kew Bulletin 61: 37-53.

Jablonski, E. 1969. Notes on Neotropical Euphorbiaceae. 4. Monograph of the genus Actinostemon. Phytologia 18: 213-240.

Judd, W.S., Campbell, C.S., Kellog, E.A. \& Stevens, P.F. 1999. Plant Systematics: A phylogenetic approach. Sinauer Associates, Sunderland.

Kathriarachchi, H., Hoffmann, P., Samuel, R., Wurdack, K.J. \& Chase M.W. 2005. Molecular phylogenetics of Phyllanthaceae inferred from five genes (plastid atp, matK, $3 n d h F, r b c L$, and nuclear PHYC). Molecular Phylogenetics and Evolution 36: 112-134.

Lucena, M.F.A. 2009. Diversidade de Euphorbiaceae sensu lato no Nordeste do Brasil. Tese de Doutorado, Universidade Federal de Pernambuco, Recife.

Macbride,J.F. 1951. Flora of Peru: Euphorbiaceae. Field Museum of Natural History 13: 1-200.

Melo, A.L. 2005. Revisão de Sebastiania Spreng. sensu stricto (Euphorbiaceae-Hippmaneae). Tese de Doutorado, Universidade Federal Rural de Pernambuco, Recife.

Melo, A.L. \& Sales, M.F.2008. O gênero Cnidoscolus Pohl (Crotonoideae-Euphorbiaceae) no Estado de Pernambuco-Brasil. Acta Botanica Brasilica 22: 806-827.

MMA. 2002. Avaliação e ações prioritárias para conservação da Biodiversidade da Caatinga. Ministério do Meio Ambiente, dos Recursos Hídricos e da Amazônia Legal, Universidade Federal de Pernambuco, Conservation International do Brasil e Fundação Biodiversitas, Brasília.
Müller, J. 1873. Euphorbiaceae. In: C.F.P. Martius \& A.G. Eichler (eds.). Flora Brasiliensis. Typografia Regia, Monachii, v. 11, pp. 1-750.

Pax, F. \& Hoffmann, K. 1922. EuphorbiaceaePhyllanthoideae-Phyllantheae. In: A. Engler, Das Pflanzenreich IV. 147:182-185.

Radcliffe-Smith, A. 2001. Genera Euphorbiacearum. Royal Botanic Gardens, Kew.

Santos Filho, F.S. 2000. A Família Euphorbiaceae Juss. no Parque Estadual Zoobotânico na cidade de Teresina, Piauí-Brasil. Dissertação de Mestrado, Universidade Federal de Pernambuco, Recife.

Sátiro, L.N. \& Roque, N. 2008. A família Euphorbiaceae nas caatingas arenosas do médio rio São Francisco, BA, Brasil. Acta Botanica Brasilica 22: 99-118.

Secco, R.S. 2008. Sinopse das espécies de Croton L. (Euphorbiaceae) na Amazônia brasileira: um ensaio taxonômico. Museu Paraense Emílio Goeldi, Belém.

Simpson, M.G. 2006. Plant systematics. Elsevier Academic Press, Amsterdam.

Soltis, D.E., Soltis, P.S., Endress, P.K. \& Chase, M.W. 2005. Phylogeny and evolution of Angisosperms. Sinauer Associates, Sunderland.

Souza, V.C. \& Lorenzi, H. 2006. Botânica sistemática: Guia ilustrado para identificação das famílias de Fanerógamas nativas e exóticas no Brasil, baseado em APG II. Instituto Plantarum, Nova Odessa.

Steinmann, V.W. \& Porter, J.M. 2002. Phylogenetic relationships in Euphorbieae (Euphorbiaceae) based on ITS and ndhF sequence data. Annals of the Missouri Botanical Garden 89: 453-490.

Steinmann, V.W. 2003. The submersion of Pedilanthus into Euphorbia (Euphorbiaceae). Acta Botanica Mexicana 65: 45-50.

Thiers, B. 2009. Index herbariorum. http://sciweb. nybg.org/science2/IndexHerbariorum.asp. (acesso em 15.12.2009).

Webster, G.L. 1984. A revision of Flueggea (Euphorbiaceae). Allertonia 3: 259-312.

Webster, G.L. 1989. Three new species of Dalechampia (Euphorbiaceae) from Brazil. Brittonia 41: 1-9.

Webster, G.L. 1993. A provisional synopsis of the sections of the genus Croton (Euphorbiaceae). Taxon 42: 793-823.

Webster, G.L. 1994. Systematics of the Euphorbiaceae. Annals of the Missouri Botanical Garden 81: 1144. 
Webster, G.L. \& Berry, P. 1999. Wurdack, K.J., Hoffmann, P. \& Chase, M.W. Euphorbiaceae. In: J.A. Steyermark, P.E. Berry, K. Yatskievych \& B.K. Holst (eds). Flora of the Venezuelan Guayana. Missouri Botanical Garden Press, St.

Louis, pp. 72-228. 2005. Molecular phylogenetic analysis of uniovulate Euphorbiaceae (Euphorbiaceae sensu stricto) using plastid $r b c l$ and trnl-f DNA sequences. American Journal of Botany 92: 1397-1420. 